\title{
ConvoCons: Encouraging Affinity on Multitouch Interfaces
}

\author{
Michael A. Oren and Stephen B. Gilbert \\ Iowa State University, Graduate Program in Human Computer Interaction, 1620 Howe Hall, \\ Ames, IA, 50011, United States \\ \{moren, gilbert\} @iastate.edu
}

\begin{abstract}
This paper describes the design of ConvoCons, a system to promote affinity of group members working in a co-located multitouch environment. The research includes an exploratory study that led to the development of ConvoCons as well as the iterative evolution of the ConvoCon system, design tradeoffs made, and empirical observations of users that led to design changes. This research adds to the literature on social interaction design and offers interface designers guidance on promoting affinity and increased collaboration via the user interface.
\end{abstract}

Keywords: Multitouch, affinity, table computing, collaboration, virtual assembly, creativity support.

\section{Introduction}

When individuals work together for the first time they lack knowledge of one another's reputations and other elements typically useful for successful cooperation [1]. Strangers cooperating for the first time without a shared connection to facilitate introductions and establishing common ground may at first struggle to establish a level of affinity needed for productive cooperation [3][12]. Individuals seek affinity as a means to fill a need for interpersonal relationships and established affinity is necessary for sustained cooperative relationships [7][17]. We created a system, ConvoCons, as a means of helping strangers begin the process of building affinity and using cooperative strategies.

Our research intends to answer the question of whether or not a software interface can be built that can promote affinity between group members in a co-located collaborative environment. This paper presents an exploratory study that led to the ConvoCons framework and discusses the design trade-offs and iterative evaluation process that led to our current system. At this point, our research focuses on affinity creation and does not look at the length of affinity bonds created nor does it explore whether or not affinity creation through our system promotes cooperation in a competitive environment; it simply seeks to explore a low-cost method of promoting affinity within a co-located dyad where neither partner has previous knowledge of the other.

The system we created, ConvoCons, is an applied reification of Nardi's observations suggesting that incidental communication, even if unrelated to the task at hand, 
is critical to supporting productive collaborative strategies [12]. ConvoCons, defined as conversation starting icons or other visual features, are designed to serve as icebreakers and casual distractions to encourage informal discourse between new partners. This informal discourse leads to connections that aid in collaboration critical to productive collaborative strategies [12]. We believe that these affinity bonds, formed through the affinity of discussing the ConvoCons, lead to the critically important state of social cohesion [10].

To measure whether the ConvoCons approach increases affinity, a measurable definition is required. Nardi [12] defines affinity as a 'feeling of connection between people'. We have narrowed this definition to the "convergence of thoughts, actions, or ideas" and made the following assumptions for measurement purposes within a multitouch collaborative context. First, a group that lacks affinity will have group members that are more likely to work independently from one another and more likely to enforce personal space. Signs of increasing affinity include actions such as reaching into a partner's personal space. Personal space on a multitouch device is defined as the area immediately in front of an individual [14][16]. Second, joint work is also a sign of affinity as coordination is required. A leader-follower approach, with one person directing the other, may be a sign of affinity if the partners deem the work afterward as equally representing each other's ideas; the leader-follower dynamic can demonstrate that the partners understand each other's roles and skills. Third, communications indicating agreement and affirmation of actions are also indicators of a group that has acquired a degree of affinity. Fourth, high affinity groups will indicate no hesitation in close proximity working areas. Fifth, planning communications, e.g., discussion about what to make and how to make it are indicators of a group that has acquired affinity. Sixth, communication unrelated to the task is an indicator of affinity, including reading ConvoCons to one another. Simiarly, shared laughter is also an indicator of increased affinity.

Given the desire to create an interface component designed to promote affinity, three main challenges arise: the types of content to use, when to display it, and how to integrate it visually with the interface at hand. The research described below describes four phases of efforts to refine the ConvoCon design based varied approaches to addressing these challenges. Phase I is based on a study of Baseplate, a virtual block assembly application, where we discovered that dyads used the abstraction present in the interface as a means of jumpstarting collaboration on a multitouch table using the SparshUI architecture [13][15]; the interface itself served as ConvoCon. Phase 2 describes a separate ConvoCon interface layer that could be attached to applications as a means of promoting affinity. Phase 2 used news headlines that appeared as rotating circles within the middle of the multitouch device that appeared in the same color each time, had some transparency, and rotated in a circle so both participants could view it (see figures 1-4 below). Phase 3 displayed riddles and jokes (first the question, then the next ConvoCon would display the joke or punch line) and the text would flash on and off as they rotated in the center of the screen. Phase 4 ConvoCons displayed a question (either a joke or riddle) on one end of the device while displaying an answer on the other end of the device to face the participant at each end. The background color of the ConvoCon was different each time.

We conducted this study using a multitouch device as it allowed co-located collaboration where both pairs in a dyad could have equal control over the results, but unlike 
collaboration with paper on a physical table we could dynamically display ConvoCon items [2]. These initial phases of ConvoCon research takes place in a co-located environment to optimize conditions for partners to understand one another's intended actions ("social translucence") [9]. To enable two participants to collaborate, Phases 1 and 2 employed an FTIR-based 60" multitouch table [4] with participants standing. Phases 3 and 4 used a Stantum 15.4" multitouch display with participants seated.

\section{Phase 1 ConvoCons}

Phase 1 was originally designed as an exploratory study to evaluate the use of Baseplate, a collaborative block-assembly application, in a co-located and remote environment to explore the collaboration strategies used by participants. However, after running this study, the main findings led to our development of ConvoCons. We analyzed the conversations that created bonds within dyad groups that led to their collaboration on the tasks. [5] and [6] describe the need for collaborators to have a shared vocabulary of the task in terms of distributed cognition. When using Baseplate, participants needed to have a shared understanding/vocabulary of the interface to complete the tasks.

From results that showed that elements of the interface led to collaborative strategies, we were able to develop the initial framework for ConvoCons, which we hoped to use for the purpose of creating a theoretical framework to guide the future designs of co-located and remote collaboration virtual assembly environments.

\subsection{Phase 1 Methods}

Our first experimental groups consisted of five co-located dyads that used the table, having a shared physical space (the input device of the tabletop) and a shared virtual space (the Baseplate workspace) where work was performed on a 60" FTIR table [13]. The second experimental group consisted of three dyads where one participant using Baseplate on the table and the other participant using Baseplate on the Stantum-in this condition participants were located in the same room and allowed to talk with each other, but they were not allowed to look at one another's devices.

All participants were asked to reproduce a series of simple, 2D patterns using Baseplate for three tasks, with ten minutes per task, and then were given up to ten minutes to create a new pattern collaboratively for a total of four tasks. Beyond instructions on how to place, rotate, and move blocks, participants were not given any information about the interface and what each block represented. Baseplate and the patterns used for Tasks 1- 3 can be seen in Figure 1.
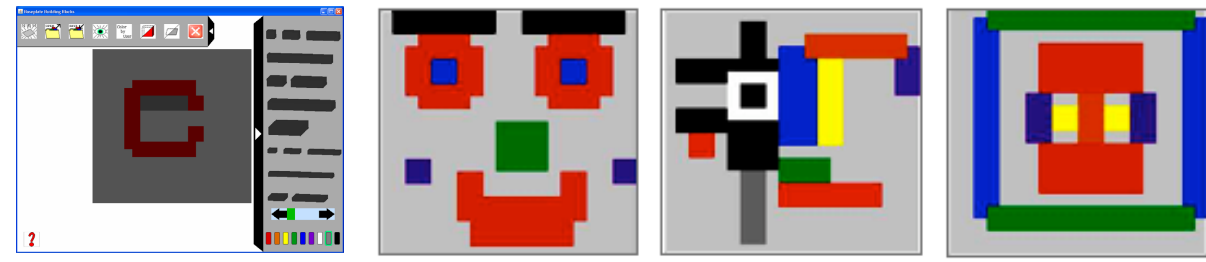

Fig. 1. Baseplate (left) and the three task patterns used in the study 
Participants' hands and conversations were video recorded during task completion. Video and audio feeds were analyzed qualitatively for strategies of collaboration, while survey data were analyzed quantitatively.

\subsection{Phase 1 Results and Discussion}

We observed a discussion that often jumpstarted the collaborative process for dyads in both focused on which block in the interface represented which block in the pattern. This discussion resulted from the ambiguity of the blocks within the interface (appearing from a 3D perspective view, whereas blocks in the pattern were in a $2 \mathrm{D}$ top-down view). The interface's ambiguity was thus a source of increased affinity. The idea that a challenging interface can lead to positive collaborative strategies may seem counterintuitive to a HCII audience, but it aligns with the MIT constructionism philosophy that participants learn precisely through such meaning making during constructive design [8]. Developing challenging interfaces intentionally, of course, would incur significant usability costs. Seeking a solution that would provide similar affinity benefits without the cognitive or usability costs, we began ConvoCons research.

Given Nardi's observations noted above on the importance to casual conversation for affinity building, the question remained of whether the key to increased affinity and effective collaboration was task-related conversation (about the interface, leading to a shared representation of the application) or whether any conversation would have helped (per Nardi).

\section{Phase 2 ConvoCons}

For Phase 2, we designed a structured ConvoCons system to promote affinity that could be used with any and all of our multitouch applications with equal effectiveness in promoting affinity. Since our original design was focused on promoting affinity for groups standing around a 60" FTIR table, we chose to make the ConvoCons round to indicate that they are intended for all individuals and to rotate them so no single user had a privileged view that would provide them ownership of the ConvoCon content. ConvoCons were circular, placed in the center of the display with a width of approximately $30 \%$ of the total table width and a $50 \%$ transparent background so users would both be forced to pay attention to it and able to continue working while displayed. Since ConvoCons were intended to serve as icebreakers, the first touch to occur on the multitouch device triggered the display of the first ConvoCon. This first iteration used the day's news headlines as an informal icebreaker to promote affinity. ConvoCons appeared during the first 15 minutes of interaction at 1.5-minute intervals. The 15-minute time limit was set to allow their use as icebreakers but prevent participants from being distracted during the entire course of the task. The 1.5-minute interval was set as a sufficient amount of time for the previous ConvoCon to make a full rotation while also providing one minute of uninterrupted work time for participants.

In creating this initial design for ConvoCons, certain compromises were made based on choices in design tradeoffs. The most significant of these compromises was the fact that the design risked annoying and alienating users by appearing in the center of their work area; however, we chose to do this because it provided equal access to 

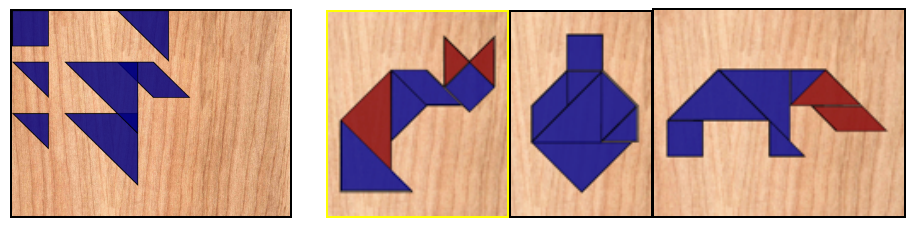

Fig. 2. The virtual tangram application and the three patterns

all collaborators and because we wanted to force users to attend to the ConvoCons so we could observe the effects of ConvoCons on affinity building. This was particularly an issue of possible user frustration since ConvoCons could not be moved out of the way or made to disappear before they had completed a full rotation-this was intended to prevent one user from taking control and dominating the ConvoCons, which would prevent them from serving as a means of promoting affinity at a level of equality within the group. In addition, headlines required minimal for reading, though they required users to attend more fully to reading them than to and image or color. Headlines also had the issue that three to ten words often provide very little information to begin a discussion about a topic if no participants are familiar with the story.

In order to evaluate the efficacy of this design, an initial pilot test of the ConvoCons system was conducted. This pilot took place on a 60" FTIR table using our Tangrams application as seen in Figure 2. Tangrams offers puzzles that require users to combine smaller geometric shapes into a larger geometric shape. This application was chosen as the initial test bed for the ConvoCons system due to the graphical simplicity and clarity of the application, which would ensure minimal confusion from abstractions of interfaces that could serve as an additional means of affinity building. Participants were given instructions on how to rotate, drag, and flip the seven shapes that make up tangram puzzles. Participants were then asked to complete three tangram puzzles with the solutions seen in Figure 2 and told they had up to ten minutes per puzzle. After participants completed all three puzzles they were given up to ten minutes to create anything they wanted.

\subsection{Phase 2 Results}

Phase 2 ConvoCons were evaluated with three dyads with each dyad containing one male and one female participant and a mean age of 27. All participants in this phase had previous experience with multitouch and four of the six individuals reported their sociability as not very social, defined in our Likert scale as preferring tight groups while two reported it as highly social, defined in our Likert scale as being comfortable with talking to strangers. The two highly social participants were part of the same dyad, this dyad rated each other as acquaintances. The other two dyads rated familiarity with their partner as "seen around" and "never met," respectively.

This initial iteration of ConvoCons failed almost entirely to promote affinity in a manner that would not be invasive to users. Users sometimes read the first headline and then quickly came to ignore all subsequent content of ConvoCons. Users still attended to the ConvoCons on occasion after the initial headline, but conversations about the ConvoCons were focused on how to get rid of them and about how annoying they were while trying to complete the puzzles. In unstructured interviews after 
the tasks were completed, participants were unable to remember any headline in its entirety and only had a vague notion of one headline at most. All participants stated that they found the ConvoCons to be annoying and distracting and described them with such terms as "irrelevant" and "uninteresting." Two participants, each in different dyads, noted that they felt a sense of bonding over the ConvoCons in the annoyance they shared with their partner as they tried to get the ConvoCons to go away. The last of these three groups tried a modified version where the ConvoCon text flashed; however, they found it difficult to read and annoying and ignored the ConvoCons just as much as the previous groups.

\section{Phase 3 ConvoCons}

With the failure of Phase 2 ConvoCons came the need to tweak the ConvoCon system in hopes we could still promote affinity through their use. Phase 3 ConvoCons incorporated the same design elements as Phase 2 ConvoCons but were made harder to become habituated to by having each ConvoCon appear with a random colored background. In this iteration, the headline text was replaced with riddles or jokes with the question displayed and then the next ConvoCon displaying the answer or punch line. This created the tradeoff that this iteration was very culturally grounded so its global use would be highly limited. In addition, riddles and jokes tended to be significantly longer than headlines, so users had to devote more time to read and process the text to converse about it. However, unlike headlines participants these required no additional information in order to fully understand the information. Since the first riddle and joke portion of the ConvoCon was posed as a question, it provided a potential point for users to discuss it to try to figure out the answer or punch line before receiving it from the system.

The procedures for evaluating Phase 3 ConvoCons were similar to that of Phase 2 in that participants received training of the basic functionality of the system and were then asked to complete three patterns, the same three that were used for Phase 2, and were then given up to ten minutes to create any pattern they chose using the tangram pieces. However, unlike Phase 2, participants in this phase were not told of a time limit to complete the puzzles as some results in Phase 2 raised concern that placing a time on puzzle completion may bias participants to be focused on reducing the time spent on non-task oriented items like reading ConvoCons text and talking to one another. This concern arose from the unstructured interviews of Phase 2 where one participant noted that her reason for ignoring the ConvoCons was a feeling that she needed to complete the task as quickly as possible given the time constraint.

\subsection{Phase 3 Results}

Phase 3 involved six dyads recruited from the undergraduate psychology department. Our observations indicated significant confusion of participants when the first ConvoCon appeared with one participant remarking "I didn't know there'd be a quiz." It was also observed that due to participants' focus on the task that by the time the answer arrived they often had forgotten the question. Generally, conversations between these dyads were muted, both in terms of conversations around ConvoCons and conversations about the task, with conversations focusing mostly on issues where the 
solution they were independently working on was found not to work. In unstructured interviews after the tasks, two of the six groups paid significant attention to the ConvoCons with a member from group 2 stating they originally paid more attention to the ConvoCon text than they did the task. One of the two groups that attended to the ConvoCons had a modified version where two ConvoCons were displayed simultaneously without rotation with one partner receiving the question and the other the answer. The group with this slight modification recalled the greatest number of ConvoCons ( 3 of 10 ). This group was also the only of the six groups to read aloud a ConvoCon beyond the first one. The group with the modified Phase 3 ConvoCons was composed of a mixed sex dyad who had never met and both self-reporting a preference for "tight groups."

\section{Phase 4 ConvoCons}

With the promising results from the slightly modified Phase 3 ConvoCons, we formalized the design modifications for two ConvoCons on opposite sides of the multitouch display with fixed orientation toward one user, similar to the placement of numbers or letters on playing cards. This approach provided the tradeoff that this version of ConvoCons biases interaction and affinity promotion toward two individuals rather than a group. No other visual changes were made to the ConvoCons.

The procedure used was changed slightly for Phase 4 ConvoCons evaluation in order to further reduce user focus on the tasks to promote user attendance to the ConvoCons. This change was to remove the training on the Tangram application and instead provide five minutes for participants to play with the system, during which ConvoCons appeared from first touch and at minute and a half intervals. This playtime had the additional advantage in that it allowed us to evaluate the intuitiveness of the gestures employed in the application. The decision to make this change came from an observation of a tour group to which we demoed the ConvoCons-enabled Tangram application. We observed a user on the answer side covering it up while another user read the question, which suggested to us that participants may need a similarly relaxed setting in order to make use of the ConvoCons as an affinity building mechanism. Given the power of authority and the tendency to conform to assigned roles demonstrated by Milgram and Zimbardo [11][18], participants may have been strongly focused on the puzzle tasks by 1) hearing our experimenter conduct training on the tasks and 2) knowing that they would receive a departmental research credit for participating in the study. The playtime was designed to lessen these influences.

We also ran participants that used Tangrams without ConvoCons but with the playtime in order to ensure that observations of affinity were a product of ConvoCons and not the playtime.

\subsection{Phase 4 Results}

Phase 4 was evaluated using ten dyads using a ConvoCons enabled version of Tangrams and nine dyads with ConvoCons turned off. Observations of users suggested that playtime does have a role in users attending to ConvoCons as all groups attended to at least one ConvoCon during this playtime and all but one ConvoCon group chose to use the entire five minutes of the playtime although all but two groups had learned all gestures 
within the first two minutes of training. In contrast, only three of the nine dyads working without ConvoCons used the entire five minutes of playtime, most stopping after roughly three minutes, one stopping after just over one minute, and others asking the researcher to advance to the task or sitting in awkward silence staring at the researcher or at their own hands until being asked if they wanted to start the puzzles.

In unstructured interviews after the tasks were completed, dyads were able to remember at least three ConvoCons, both the general content and specifics, the dyads also stated that during the playtime the ConvoCons were not distracting or annoying although they were at first confused what they were and why they were there. Reaction to the ConvoCons during task time were similar to those in previous iterations where they were often ignored, although some groups continued to pause work to read over and have a shared laugh over a joke or try to solve a puzzle - this most often occurred when a ConvoCon appeared while a dyad was having difficulty solving a puzzle. One group, in commenting on the ConvoCons, responded, "[ther were] maybe not so much for getting to know each other, but for creating conversation." Another group stated that the ConvoCons probably made them talk more than they would have without them; however, they also felt the ConvoCons were irrelevant and distracting.

\section{Conclusions}

Phase 4 ConvoCons indicate that it is possible to create a layer on top of the interface that enables users unfamiliar with one another to build affinity. Efforts to code and quantitatively compare the levels of affinity for each of the groups in Phase 4 are underway to determine the magnitude of the effects of ConvoCons. Future work looks to expand ConvoCons beyond dyads to small groups in addition to looking at the effects of ConvoCon-encouraged affinity when a reward structure is present that would result in a level of competition between participants. We also hope to explore the use of ConvoCons as a way of building affinity among remote collaborators.

Acknowledgements. We thank Cole Anagnost, Thomas Niedzielski, and Desirée Velázquez for developing the initial Baseplate application. We also thank Jay Roltgen, Prasad Ramanhally, Ashley Polkinghorn, and Ankit Patel for their development efforts of the ConvoCon code base and the Tangram application. This research was performed as part of a Research Experience for Undergraduates sponsored by NSF (IIS-0552522). Additional funding was provided by the Air Force Research Lab and the Grow Iowa Values Fund.

\section{References}

1. Bolton, G., Katok, E., Ockenfels, A.: Cooperation among strangers with limited information about reputation. Journal of Public Economics 89, 1457-1468 (2005)

2. Buxton, W., Hill, R., Rowley, P.: Issues and techniques in touch-sensitive tablet input. In: Proc. of SIGGRAPH 1985, pp. 215-224 (1985)

3. Convertino, G., Mentis, H., Rosson, M., Carroll, J., Slavkovic, A., Ganoe, C.: Articulating common ground in cooperative work: content and process. In: Proc. of CHI 2008, pp. 1637-1646 (2008) 
4. Dohse, K., Dohse, T., Still, J., Parkhurst, D.: Enhancing Multi-user Interaction with MultitouchMultitouch Tabletop Displays Using Hand Tracking. In: Proc. of Tabletop 2008, pp. 297-302 (2008)

5. Fischer, G., Arias, E., Carmien, S., Eden, H., Gorman, A.: Supporting Collaboration and Distributed Cognition in Context-Aware Pervasive Computing Environments. In: Proc. of HCIC 2004 (2004)

6. Hollan, J., Hutchins, E., Kirsh, D.: Distributed cognition: toward a new foundation for human-computer interaction research. TOCHI (2000)

7. Honeycutt, J., Patterson, J.: Affinity Strategies in Relationships: The role of gender and imagined interactions in maintaining college roommates. Personal Relationships 4, 35-46 (1997)

8. Kafai, Y., Resnick, M.: Constructionism in Practice, 1-8 (1996)

9. Kellogg, W., Erickson, T.: Social Translucence, Collective Awareness, and the Emergence of Place. In: Proc. of CSCW 2002 (2002)

10. King, J., Star, S.: Conceptual Foundations for the Development of Organizational Decision Support Systems. In: The Proceedings of the Hawaii International Conference on Systems Science, pp. 143-151 (1990)

11. Milgram, S.: Obedience to Authority. Tavistock Publication, Londong (1974)

12. Nardi, B.: Beyond Bandwidth: Dimension of Connection in Interpersonal Communication. In: Proc. of CSCW 2005, pp. 91-129 (2005)

13. Ramanahally, P., Gilbert, S., Anagnost, C., Niedzielski, T., Velázquez, D.: Creating a Collaborative Multi-Touch Computer Aided Design Program. In: Proc. of WinVR 2009 (2009)

14. Scott, S., Grant, K., Mandryk, R.: System guidelines for co-located, collaborative work on a tabletop display. In: Proc. of the Eighth European Conference on Computer-Supported Cooperative Work (2003)

15. Sparsh UI (2008), http://code.google.com/p/sparsh-ui/ (retrieved September 7, 2008)

16. Tuddenham, P., Robinson, P.: Distributed Tabletops: Supporting Remote and MixedPresence Tabletop Collaboration. In: Proc. of IEEE International Workshop on Horizontal Interactive Human-Computer Systems 2007, pp. 19-26 (2007)

17. Whittaker, S.: Theories and Models in Mediated Communication. In: Graesser, A. (ed.) The Handbook of Discourse Processes. Lawrence Erlbaum, Cambridge (2003)

18. Zimbardo, P., Maslach, C., Haney, C.: Reflections on the Stanford Prison Experiment: Genesis, Transformation, Consequences. In: Blass, T. (ed.) Obedience to Authority: Current Perspectives on the Milgram Paradigm, pp. 193-238. Lawrence Erlbaum Associates, Mahwah (2000) 